American Journal of Animal and Veterinary Sciences 7 (2): 104-111, 2012

ISSN 1557-4555

(C) 2012 Science Publications

\title{
Progress in Relevant Growth Factors Promoting the Growth of Hair Follicle
}

\author{
Wang, J.M. and J.T. Zhang \\ Laboratory Animal Center, Zhengzhou University, Zhengzhou, P R China
}

\begin{abstract}
Hair is a protective appendage on the body that is considered accessory structure of the integument. Hair follicle development takes place during fetal skin development and relies on tightly regulated ectodermal-mesodermal interactions. The morphological changes of the hair cycle have been very clear that hair morphogenesis and epidermal development are orchestrated by an array of growth factors. In this review, we summarize the major growth factors involved in promoting growth of hair follicles.
\end{abstract}

Key words: Hair follicle, growth factor, Dermal Sheath (DS), Inner Root Sheath (IRS), Dermal Papilla (DP), Outer Root Sheath (ORS), Hair Shaft (HS)

\section{INTRODUCTION}

The skin is the body's largest organ, covering the body surface and in contact with the outside world. It is a composition by the keratinized stratified epidermis and collagen-rich dermal connective tissue, which contains a subsidiary organ of the hair follicles, sebaceous glands, sweat glands and nails, derived from the epidermis. Skin and its appendages provide a protective barrier essential for animal survival.

Hair is a primary characteristic of mammals and exerts a wide range of functions including thermoregulation, physical protection, sensory activity and social interactions (Schneider et al., 2009). The hair follicle is a unit formed by interaction of the epithelial cells, nerve endings and stromal cells (Lee, 2011). its occurrence and maintenance related to different cell subsets from more than twenty types of different germ layers, whose structures and function are very complex. These cells grow in the Dermal Sheath (DS), Inner Root Sheath (IRS), Outer Root Sheath (ORS), Hair Shaft (HS) and Dermal Papilla (DP). Developmental biology considered that hair follicle is a significative model of interaction of epithelium and mesenchyma (Yang and Cotsarelis, 2010). Meanwhile, the hair follicle is the only structure with remaining morphogenesis capacity and self-regeneration of mature mammals and plays an important role in its cyclical growth process.

Studies have shown that hair follicles can produce a variety of signal transduction molecules (short peptides or small proteins), soluble molecules (cytokines) or cell surface receptors and proceed with intercellular signal transduction and regulate the hair follicle cyclical growth with the help of these molecules. Development of hair follicles begins towards the end of the first trimester of pregnancy and is controlled by Epidermal-Mesenchymal Interaction (EMI), which is a signaling cascade between epidermal and mesenchymal cell populations (Yoo et al., 2010). Hair follicles undergo a cycle of developmental events: the growth period (anagen), regression period (catagen) and quiescence period (telogen) (Jiang et al., 2010). This process is a complex and orderly cycle affected by many factors, while these effects must be coordinated through the relevant growth factor or hormone as an intermediary secreted by the body. Therefore, the growth factor, as an important link of coordination genes secreted by the body and the environment, plays a vital role in the growth and regulation of the hair follicle.

The morphological changes of the hair cycle have been very clear while the changes in the molecular signaling and channels in the regulation of hair follicles between phases are in a constant study. Therefore, looking for the relevant growth factor affecting hair follicle growth has been the target of numerous studies. In recent years, it has been reported that the growth factors, such as EGF, TGF- $\beta$, IGF-1, HGF, KGF, VEGF and so on, participate in the hair follicle cycle regulation and promote hair growth. In this review, we summarize their progress at home and abroad in the past few years.

Growth factors:

The Epidermal Growth Factor (EGF) family: The EGF family of growth factors comprises EGF, Transforming Growth Factor-alpha (TGF- $\alpha$ ), Heparin-

Corresponding Author: Zhang, J.T., Laboratory Animal Center, Zhengzhou University, Zhengzhou, P R China 
American J. Animal \& Vet. Sci., 7 (2): 104-111, 2012

Binding EGF-like growth factor (HB-EGF), Amphiregulin (AR), Betacellulin (BTC), Epiregulin (EPR), Epigen (EPG) and Neuregulins (NRGs) (Wilson et al., 2009). EGF family members activate an intracellular signaling cascade upon binding to their receptors, collectively known as the ErbB family. The ErbB family comprises ErbB1 (also universally referred to as EGFR), ErbB2, ErbB3 and ErbB4. Signals originating from ErbB1 play crucial roles in the development of epithelial tissues. Human epidermal keratinocytes express ErbB1, ErbB2 and ErbB3, but not ErbB4.

ErbB1 is the prototype of the Receptor Tyrosine Kinase (RTK) family and is a transmembrane glycoprotein, including the extracellular ligand binding domain, transmembrane and intracellular region (this region has intrinsic tyrosine kinase activity) (Zhou and Agazie, 2012). ErbB1 participates in the regulation of cell metabolism, proliferation, migration and differentiation of physiological processes, whose signaling is necessary for proper development and tissue homeostasis whereas its dysregulation rapidly results in defects in cellular proliferation and differentiation. ErbB1 ligands are very important in the skin (Sugawara et al., 2010). TGF- $\alpha$ and EGF bind exclusively to ErbB1 and are related autocrine/ paracrine growth factors that activate ErbB1 to regulate the balance between keratinocyte proliferation and differentiation (Cheng et al., 2010). ErbB1 and its ligands represent one of the most powerful and complex signaling networks in higher vertebrates. In this pleiotropic system that exerts an unusually wide array of diverse bioregulatory functions, several peptide growth factors promote the homo-or heterodimerization and subsequent autophosphorylation of a family of tyrosine kinase receptors. Consequently, adaptor proteins and enzymes initiate signaling cascades, culminating in biological outcomes ranging from cell division to cell death, differentiation, or malignant transformation (Gusterson and Hunter, 2009).

Studies have shown that ErbB1 as an important growth factor receptor and its ligand EGF exist in ORS of the mature hair follicle and play an important role in the regulation of hair follicle growth cycle. EGF binds to ErbB1 and participates in regulating DNA synthesis rate of outer root sheath cells and hair bulb cells of hair follicle, further induces the differentiation of the hair bulb cells, making the external root sheath cells differentiate, so that the hair follicles enter regression period in advance from the growth period (Alexandrescu et al., 2009). In the early stages of hair follicle growth, EGF and TGF- $\alpha$ can inhibit hair follicle formation, which are great related with ErbB1.
Transforming Growth Factor- $\beta$ (TGF- $\beta$ ): The TGF- $\beta$ super family is a large family of proteins that comprises the various isoforms of TGF- $\beta$, Bone Morphogenetic Proteins (BMPs), the nodals, activins and inhibins, antiMullerian hormone and many other structurally related factors (Ehrlich et al., 2012; Pohlers et al., 2009). The TGF- $\beta$ family consists of three members, TGF- $\beta 1$, TGF- $\beta 2$ and TGF- $\beta 3$ in mammals (Arjaans et al., 2012) TGF- $\beta$ s are secreted with a dimeric Latency-Associated Peptide (LAP) in an inactive form called Small Latent Complex (SLC) and dissociation from LAP releases the approximately $25 \mathrm{kDa}$ mature polypeptides (Hinck, 2012). Latent TGF- $\beta$ Binding Proteins (LTBPs) are a family of fibrillin-like molecules that are covalently linked to SLC. In addition to acting as matrix components, LTBPs regulate TGF- $\beta$ bioavailability and activity by facilitating latent TGF- $\beta$ secretion, mediating latent TGF- $\beta$ targeting to the ECM and regulating latent TGF- $\beta$ activation ( $\mathrm{Zi}$ et al., 2012). TGF- $\beta$ isoforms regulate multiple biological processes including cell proliferation, extracellular matrix synthesis, angiogenesis, immune response, apoptosis and differentiation (Lampropoulos et al., 2012).

TGF- $\beta$ plays an important role in controlling hair growth cycle, mainly regulating the initiation and stop of catagen phase (Oshimori and Fuchs, 2012). In mature hair follicles, TGF- $\beta 1$ mRNA is expressed in the inner hair root sheath, TGF- $\beta 2$ mRNA is expressed at the end of the epithelium, while TGF- $\beta 3$ in the neighboring district of the outer root sheath (Inui and Itami, 2011). Recent studies have found that expression of TGF- $\beta 1$ in hair follicle cells have relations with epidermal repair (Liang et al., 2012). These studies suggest that TGF- $\beta$ signal transduction not only controls the initiation of catagen phase, but also participates in many other physiological activities, which is an extremely complex process.

Smads are main intermediary of TGF- $\beta$ superfamily signal transduction. In all Smads, only loss of Smad4 in keratinocytes or overexpression of Smad7 do abnormal growth and development of the epidermis and hair follicles appear. Smad4 is mainly mediated by BMP signaling and thus affect the differentiation of hair follicles, while Smad7 significantly affect the differentiation and development of hair follicles via blocking TGF- $\beta$ /Activin/BMP pathway and inhibiting the WNT/calcium angiotensin ( $\beta$-catenin) signal (Lan and Yang, 2010) Although Smad4 is required for the Smad signal transduction, it was reported that when Smad4 was inhibited, transcriptional activity of receptor- regulated Smad (R-Smad) still could be detected. In the absence of Smad4, Smad2 and Smad3 still entered into the nucleus and further combined with 
of the target gene (Perna et al., 2012), which also showed that TNF- $\beta$ signal transduction had another path and indicated the complexity in regulating signal transduction of growth and development of the hair follicle. In addition, Smad4 gene knockout mice blocked the differentiation and periodic replacement of hair follicles, showing progressive hair loss. Furthermore, proliferation acceleration of the basal layer keratinocytes and epidermal of mutant mice affected the expression of cyclin-dependent kinase inhibitor p21 as well as squamous cell carcinoma appeared in the skin (Tang and Zhang, 2011).

Hepatocyte Growth Factor (HGF): Hepatocyte growth factor (HGF, also referred to as scatter factor) is a growth factor with stimulating primary liver cell growth and DNA synthesis from a hepatectomy rat serum and consists of a $69 \mathrm{kDa} \alpha$-chain and a $34 \mathrm{kDa} \beta$ chain (Mizuno and Nakamura, 2012). HGF was initially characterized as a powerful mitogen for hepatocytes and stimulator of epithelial cell dissociation (Lee et al., 2011). HGF with initial secretion is a non-active singlechain form and then change into the active heterodimer with the help of the serine protease HGF activator, which stimulates the growth of hepatocytes and regulates the cell proliferation, migration, survival, angiogenesis and invasion of many cells (including epithelial, vascular endothelial and some stromal cells). These diverse biological effects are mediated by stimulating the activity of its high-affinity transmembrane tyrosine kinase receptor, c-Met, a protooncogene product expressed in epithelial cells (Zhou et al., 2011; Zippel et al., 2010).

HGF can regulate the interaction between the dermal papilla cells and epithelial keratinocytes cells. Proliferation, differentiation and apoptosis of keratinocyte cells and epithelial-mesenchymal interactions, each step of them constitute an important part of hair follicle morphogenesis (Kikkawa et al., 2009). The hair follicle cycle seems to be regulating by the different types of local growth factors and their receptors and the change of balance between them may affect the hair follicle cycle (Xie et al., 2012). During the formation of a variety of mammalian organs, HGF and its receptor c-Met are required. The evidence also proved that morphogenesis of hair follicle is related to HGF and c-Met (Trusolino et al., 2010).

HGF can induce the formation of blood vessels around the hair follicles. Mecklenbury found a large number of blood vessel formation with the histomorphometry surgery and double immunohistology and a lot of proliferative vascular endothelial cells by immunofluorescence staining in the early growth of hair follicles in adult C57BL/6 mice (Sayama et al., 2010). In the hair follicle cycle, the expression of HGF and its receptor c-Met was required and HGF expressed only by the dermal papilla in the early growth stage of hair follicle. HGF/SF and c-Met had pro-angiogenic effect and promoted the expression of Vascular Endothelial Growth Factor (VEGF). It was believed that HGF and VEGF played an important role in the blood vessel formation of the hair follicle growth (Xu et al., 2011). In addition, it suggested that HGF could promote the hair follicles growth of newborn mice and the hair follicle would become longer and larger in histology when the mice was injected HGF in the dorsal skin subcutaneously (Fushimi et al., 2011). Lee showed that epithin/matriptase (Epi/MTP) which played a significant role in mammary epithelial cell growth and morphogenesis participated in mammary epithelial growth and modeling through activation of pro-HGF (Lee et al., 2010a).

Keratinocyte Growth Factor (KGF): Keratinocyte Growth Factor (KGF), a $28 \mathrm{kDa}$ polypeptide, is a member of the Fibroblast Growth Factor (FGF) family (also known as FGF-7). KGF is a potent mitogen for epithelial cells, which express a subset of FGF receptor isoforms (the FGFR2b isoforms) (Yamamoto-Fukuda $e t$ al., 2012). KGF is a mesenchymal-cell derived paracrine growth factor that specifically stimulates epithelial cell growth. It is supposed to be secreted from fibroblasts mainly in the stroma and bind to KGF Receptor (KGFR), which has been detected only on the surface of epithelial cells (Lin et al., 2010). KGFR still exists in hair follicles and is expressed in hair follicle epithelial cells and KGF in dermal papilla cells, which declares that KGF is mediated through paracrine (Belleudi et al., 2011).

Koria et al. (2011) showed KGF was expressed in normal skin and was induced upon injury. Furthermore, they indicated that KGF was predominantly expressed in stromal cells below and at the edge of the wound and that KGFR was detected in the epidermis. They concluded that a KGF-mediated paracrine interaction may be important for the migration and proliferation of epidermal keratinocytes (Koria et al., 2011). It was also indicated that KGF had marked effects on the epithelialization and healing of wounds (Macleod and Havran, 2011). Richardson et al. (2009) proved that receptors for EGF and KGF underwent marked downregulation in hair follicle placodes from multiple body sites, whereas the expression of endogenous ligands persisted throughout hair follicle initiation. When skin from the sites of primary pelage and whisker follicle development was exposed to increase levels of 
two ectopic EGFR ligands and the FGFR2 (IIIb) receptor ligand KGF, follicle formation was inhibited in a time- and dose-dependent manner. Li et al. (2009) found mechanical wounding induced ligandindependent KGFR activation and internalization. KGFR internalization and phosphorylation was associated with ROS generation along the wound edge and scavenging of ROS with NAC inhibited KGFR phosphorylation (Li et al., 2009). In addition, KGF stimulated proliferation and differentiation of early progenitor cells within hair follicles and sebaceous glands in the wound bed and adjacent dermis. There was a transient but highly significant increase in specific labeling of cycling cells in both basal and suprabasal layers that extended into the spinous layer of the regenerating epidermis. The result suggested that KGF might induce specific epithelial progenitor cell lineages within the skin to proliferate and differentiate and thus might be a critical determinant of regeneration of skin (Radek et al., 2009).

Insulin-like Growth Factor (IGF): Insulin-like Growth Factor (IGF)-I and -II, also referred to as somatomedin $\mathrm{C}$ and somatomedin $\mathrm{A}$, respectively, are peptides that produce both mitogenic and insulinlike effects. Their effects are mediated by the insulin receptor and IGF receptors (IGF-IR and IGF-IIR). IGF-I and IGF-II bind IGF-IR with high affinity; IGF-IR binds insulin with low affinity. IGF-IIR binds IGF-II with high affinity and IGF-I with low affinity and does not bind insulin.

IGF-I is a multifunctional regulatory peptide that shares structural homology with proinsulin (Kang et al., 2012). IGF-I, via binding to IGF-I receptors, regulates cell proliferation, differentiation and survival (Forbes $e t$ al., 2012). Studies have shown that a large number of IGFR-1 exist in stromal mother cell of the hair bulb close to the dermal papilla, as well as in granulosa cells of the germinal layer of the outer root sheath (Ahn et al., 2012). The dermal papilla could produce large amounts of IGF-1, IGFR-1 and IGFBPs and fibroblasts and granular layer keratinocytes of hair follicle outer root sheath in the dermis also could secrete IGF-1 while the mother cells of the hair bulb matrix couldn't, which illustrated that IGF-1 arising from dermal papilla had a paracrine role in growth of the mother cell of hair bulb matrix and the granulosa cell of germinal layer of outer root sheath (Zhang et al., 2011).

Exogenous IGF-1 not only could contribute to the increase in DNA synthesis and keratinocyte proliferation of human skin in vitro (Reznikova et al., 2010), but also could promote hair follicle growth in vitro, affecting the development of hair follicles form
(Hwang et al., 2012). When exogenous IGF-1 was added into dermal papilla cultured in vitro, the level of Protein Kinase C (PKC) didn't change, indicating that cellular signal transduction mediated by IGF-1 didn't exist in the dermal papilla. This further explained that exogenous IGF-1 in promoting hair follicle growth acted on other cells but not the dermal papilla and proved again that IGF-1 secreted by the dermal papilla played a paracrine role in division, proliferation, differentiation of the hair follicle cell. Experiments in vivo showed that either systemic or local skin injection of IGF-1 could not significantly change the growth of wool, however, IGF-1 was essential to maintain hair growth, especially in the early stages of the hair cycle, which could prevent hair follicles from entering the catagen prematurely (Zhao et al., 2011). Overexpression of IGF-binding protein 5 (IGFBP-5) in the human hair xenografts obtained from straight- and curly-haired individuals was found to result in the decreased expression of several extracellular matrix proteins and disassembly of adhesional junctions, resulting in twisted hair shafts as well as an unusual deposition of hair cuticle that may be derived from the disturbance of normal proliferation and differentiation (Sriwiriyanont et al., 2011).

Vascular Endothelial Growth Factor (VEGF): VEGF is a homodimeric, heparin-binding glycoprotein that promotes endothelial cell proliferation and migration. It exists as four isoforms, produced through alternative splicing. VEGF binds to VEGF receptor-1 (VEGFR-1, also known as Flt-1) and VEGFR-2 (also known as KDR/Flk-1). Epidermal keratinocytes secrete biologically active VEGF in vitro, while cultured epidermal sheets produce large amounts of VEGF (Li et al., 2012). In addition, VEGF widespread exists in many organs in the body of humans and animals, which is essential inducible factor in other physiological and pathological angiogenesis and plays a local regulatory role (Lin et al., 2012)

VEGF is a key factor in promoting angiogenesis, as well as influencing diverse cell functions including cell survival, proliferation and the generation of nitric oxide and prostacyclin (Ford and Amore, 2012) The perifollicular capillary network is coupled to the hair cycle, increasing during the anagen phase and then regressing during the catagen and telogen phases (Lee et al., 2010b). Transgenic overexpression of VEGF in outer root sheath keratinocytes of hair follicles strongly induced perifollicular vascularization and led to accelerate hair regrowth after depilation. Moreover, VEGF as a major mediator of hair follicle growth and cycling could promote hair growth and increase hair 
follicle and hair size (Liang et al., 2012). Domingo et al. (2010) confirmed that in vivo biological importance of epidermis-derived VEGF for cutaneous angiogenesis in a transgenic mouse model, using the keratin 14 promoter to selectively target expression of murine VEGF164 to basal epidermal keratinocytes and to follicular keratinocytes of the outer root sheath of the hair follicle.

Other growth factors: A number of studies of this mouse model have demonstrated that sensory and autonomic nerves adjacent to hair follicles and a variety of neuropeptides/neurotrophins and inflammatory cytokines are involved in the progression of hair cycling. Among them, Nerve Growth Factor (NGF) has attracted special interest recently. It has been reported that NGF and its precursor proNGF and their receptors, tyrosine kinase A (TrkA) and p75, are expressed in the epidermis and hair follicles of mouse skin and influence hair follicles cycling in skin organ culture (Shirakata, 2010).

The activity of keratinocytes in the hair follicle is regulated by signals from a specialized mesenchymal niche, the dermal papilla. Among the genes differentially expressed between mutant and wildtype dermal papilla, FGF-7, FGF-10 and IGFbp5 stand out as likely mediators of the proliferationpromoting activity of the dermal papilla during anagen. FGF-7 expression increases in the dermal papilla prior to anagen onset and exogenous FGF-7 or FGF-10 stimulates proliferation of hair follicle keratinocytes (Greco et al., 2009). Manipulations that impair the function of the FGF7/10 receptor in keratinocytes result in sparse, thin hair (EnshellSeijffers et al., 2010).

\section{CONCLUSION}

Hair formation and regulation of the hair cycle are highly complex processes. It is found that a large number of growth factors and their receptors can regulate growth and development of hair follicle, the changes of their distribution status, expression level even structure can have some impacts on growth and development of hair follicle, making abnormal change.

However, the mechanism of the growth factor is not very clear. It is generally believed that corresponding receptors of cells binding to growth factor cause the conformational changes and activation of some protein kinases, then signal to the nucleus, adjusting the cell growth. Signal transduction mechanisms of growth factors relate to complex interactions, such as phosphorylation of tyrosine, serine and threonine acid residues and activation of the transcription factors, especially, tyrosine phosphorylation has very important significance. These processes don't absolutely controlled by some single growth factor and at present most of conceptual conclusions about the growth factors are inferences based on fixed position of growth factors in the hair follicle cycle or development, which may be just the initial qualitative understanding of the hair follicle growth, in fact, the main regulation is the spatial and temporal specificity and differences of the expression of growth factors or hormone genes. Obtaining related factors affecting the growth of hair follicles ultimately shall study the growth regulation of normal hair follicle or dermal papilla, as well as related factors affecting hair follicle growth and their expression level with a particular state, which is necessary for a comprehensive evaluation of the hair follicle growth control. Therefore, further study of the exact roles and mechanisms of growth genes to look for the genes and drugs with strong specificity and targeting is of great significance in the treatment of skin diseases.

\section{REFERENCES}

Ahn, S.Y., L.Q. Pi, S.T. Hwang and W.S. Lee, 2012. Effect of IGF-I on hair growth is related to the antiapoptotic effect of IGF-I and Up-regulation of PDGF-A and PDGF-B. Ann. Dermatol., 24: 26-31. PMID: 22363152

Alexandrescu, D.T., C.L. Kauffman and C.A. Dasanu, 2009. The cutaneous epidermal growth factor network: Can it be translated clinically to stimulate hair growth? Dermatol. Online J., 15: 1-1. PMID: 19379645

Arjaans, M., T.H.O. Munnink, H. Timmer-Bosscha, M. Reiss and A.M. Walenkamp et al., 2012. Transforming Growth Factor (TGF)- $\beta$ expression and activation mechanisms as potential targets for anti-tumor therapy and tumor imaging. Pharmacol. Ther., 135: 123-132. PMID: 22587883

Belleudi, F., V. Purpura and M.R. Torrisi, 2011. The receptor tyrosine kinase FGFR2b/KGFR controls early differentiation of human keratinocytes. PLoS One, 6: e24194-e24194. PMID: 21957444

Cheng, X., J. Jin, L. Hu, D. Shen and X.P. Dong et al., 2010. TRP channel regulates EGFR signaling in hair morphogenesis and skin barrier formation. Cell, 141: 331-343. PMID: 20403327

Domingo, D.S., M.M. Camouse, A.H Hsia, M. Matsui and D. Maes et al., 2010. Antiangiogenic effects of epigallocatechin-3-gallate in human skin. Int. J. Clin. Exp. Pathol., 3: 705709. PMID: 20830241 
Ehrlich, M., O. Gutman, P. Knaus and Y.I. Henis, 2012. Oligomeric interactions of TGF- $\beta$ and BMP receptors. FEBS Lett., 586: 1885-1896. PMID: 22293501

Enshell-Seijffers, D., C. Lindon, M. Kashiwagi and B.A. Morgan, 2010. beta-catenin activity in the dermal papilla regulates morphogenesis and regeneration of hair. Dev. Cell, 18: 633-642. PMID: 20412777

Forbes, B.E., P. McCarthy and R.S. Norton, 2012. Insulin-like growth factor binding proteins: A structural perspective. Front Endocrinol. (Lausanne), 3: 38-38. PMID: 22654863

Ford, K.M. and P.A. D'Amore, 2012. Molecular regulation of vascular endothelial growth factor expression in the retinal pigment epithelium. Mol. Vis., 18: 519-527. PMID: 22419845

Fushimi, T., S. Inui, M. Ogasawara, T. Nakajima and K. Hosokawa et al., 2011. Narrow-band red LED light promotes mouse hair growth through paracrine growth factors from dermal papilla. J. Dermatol. Sci., 64: 246-248. PMID: 21996311

Greco, V., T. Chen, M. Rendl, M. Schober and H.A. Pasolli et al., 2009. A two-step mechanism for stem cell activation during hair regeneration. Cell Stem Cell, 4: 155-169. PMID: 19200804

Gusterson, B.A. and K.D. Hunter, 2009. Should we be surprised at the paucity of response to EGFR inhibitors? Lancet Oncol., 10: 522-527. PMID: 19410197

Hinck, A.P., 2012. Structural studies of the TGF- $\beta$ s and their receptors-insights into evolution of the TGF- $\beta$ superfamily. FEBS Lett., 586: 1860-1870. PMID: 22651914

Hwang, K.A., Y.L. Hwang, M.H. Lee, N.R. Kim and S.S. Roh et al., 2012. Adenosine stimulates growth of dermal papilla and lengthens the anagen phase by increasing the cysteine level via fibroblast growth factors 2 and 7 in an organ culture of mouse vibrissae hair follicles. Int. J. Mol. Med., 29: 195201. PMID: 22020741

Inui, S. and S. Itami, 2011. Molecular basis of androgenetic alopecia: From androgen to paracrine mediators through dermal papilla. J. Dermatol. Sci., 61: 1-6. PMID: 21167691

Jiang, S., L. Zhao, T. Teklemariam and B.M. Hantash, 2010. Small cutaneous wounds induce telogen to anagen transition of murine hair follicle stem cells. J. Dermatol. Sci., 60: 143-150. PMID: 21071180

Kang, H.J., Y.W. Yi, H.J. Kim, Y.B. Hong and Y.S. Seong et al., 2012. BRCA1 negatively regulates IGF-1 expression through an estrogen-responsive element-like site. Cell Death Dis., 3: e336-e336. PMID: 22739988
Kikkawa, Y.S., T. Nakagawa, H. Tsubouchi, A .Ido and T. Inaoka et al., 2009. Hepatocyte growth factor protects auditory hair cells from aminoglycosides. Laryngoscope, 119: 2027-2031. PMID: 19688848

Koria, P., H. Yagi, Y. Kitagawa, Z. Megeed and Y. Nahmias et al., 2011. Self-assembling elastin-like peptides growth factor chimeric nanoparticles for the treatment of chronic wounds. Proc. Natl. Acad. Sci. USA., 108: 1034-1039. PMID: 21193639

Lampropoulos, P., A. Zizi-Sermpetzoglou, S. Rizos, A. Kostakis and N. Nikiteas et al., 2012. TGF-beta signalling in colon carcinogenesis. Cancer Lett., 314: 1-7. PMID: 22018778

Lan, Y. and X. Yang, 2010. The role of Smad signaling in vascular and hematopoietic development revealed by studies using genetic mouse models. Sci. China Life Sci., 53: 485-489. PMID: 20596915

Lee, G.S., E.J. Hong, K.S. Gwak, M.J. Park and K.C. Choi et al., 2010a. The essential oils of Chamaecyparis obtusa promote hair growth through the induction of vascular endothelial growth factor gene. Fitoterapia, 81: 17-24. PMID: 19576968

Lee, S.L., P.Y. Huang, P. Roller, E.G. Cho and D. Park et al., 2010b. Matriptase/epithin participates in mammary epithelial cell growth and morphogenesis through HGF activation. Mech. Dev., 127: 82-95. PMID: 19853659

Lee, W.J., S.E. Park and D.K. Rah, 2011. Effects of hepatocyte growth factor on collagen synthesis and matrix metalloproteinase production in keloids. J. Korean Med. Sci., 26: 1081-1086. PMID: 21860560

Lee, W.S., 2011. Integral hair lipid in human hair follicle. J. Dermatol. Sci., 64: 153-158. PMID: 21906914

Li, M., J.D. Firth and E.E. Putnins, 2009. An in vitro analysis of mechanical wounding-induced ligandindependent KGFR activation. J. Dermatol. Sci., 53: 182-191. PMID: 19111446

Li, W., X.Y. Man, C.M. Li, J.Q. Chen and J. Zhou et al., 2012. VEGF induces proliferation of human hair follicle dermal papilla cells through VEGFR2-mediated activation of ERK. Exp. Cell Res., 318: 1633-1640. PMID: 22659165

Liang, X., S. Bhattacharya, G. Bajaj, G. Guha and Z. Wang et al., 2012. Delayed cutaneous wound healing and aberrant expression of hair follicle stem cell markers in mice selectively lacking Ctip2 in epidermis. PLoS One, 7: e29999-e29999. PMID: 22383956 
Lin, C., M. Wu and J. Dong, 2012. Quercetin-4'-O- $\beta-D-$ glucopyranoside (QODG) inhibits angiogenesis by suppressing VEGFR2-mediated signaling in zebrafish and endothelial cells. PLoS One, 7: e31708-e31708. PMID: 22348123

Lin, C.B., Y. Hu, D. Rossetti, N. Chen and D. Cassarino et al., 2010. Immuno-histochemical evaluation of solar lentigines: The association of $\mathrm{KGF} / \mathrm{KGFR}$ and other factors with lesion development. J. Dermatol. Sci., 59: 91-97. PMID: 20620021

Macleod, A.S. and W.L. Havran, 2011. Functions of skin-resident $\gamma \delta \mathrm{T}$ cells. Cell Mol. Life Sci., 68: 2399-2408. PMID: 21560071

Mizuno, S. and T. Nakamura, 2012. Improvement of sepsis by hepatocyte growth factor, an antiinflammatory regulator: Emerging insights and therapeutic potential. Gastroenterol. Res. Pract., 2012: 909350-909350. PMID: 22536224

Oshimori, N. and E. Fuchs, 2012. Paracrine TGF- $\beta$ signaling counterbalances BMP-mediated repression in hair follicle stem cell activation. Cell Stem Cell, 10: 63-75. PMID: 22226356

Perna, D., G. Fagà, A. Verrecchia, M.M. Gorski and I. Barozzi et al., 2012. Genome-wide mapping of Myc binding and gene regulation in serumstimulated fibroblasts. Oncogene, 31: 1695-1709. PMID: 21860422

Pohlers, D., J. Brenmoehl, L. Loffler, C. K. Muller and C. Leipner et al., 2009. TGF-beta and fibrosis in different organs - molecular pathway imprints. Biochim. Biophys. Acta, 1792: 746-756. PMID: 19539753

Radek, K.A., K.R. Taylor and R.L. Gallo, 2009. FGF-10 and specific structural elements of dermatan sulfate size and sulfation promote maximal keratinocyte migration and cellular proliferation. Wound Repair Regen, 17: 118-126. PMID: 19152659

Reznikova, T.V., M.A. Phillips, T.J. Patterson and R.H. Rice, 2010. Opposing actions of insulin and arsenite converge on PKCdelta to alter keratinocyte proliferative potential and differentiation. Mol. Carcinog., 49: 398-409. PMID: 20082316

Richardson, G.D., H. Bazzi, K.A. Fantauzzo, J.M. Waters and H. Crawford et al., 2009. KGF and EGF signalling block hair follicle induction and promote interfollicular epidermal fate in developing mouse skin. Development., 136: 21532164. PMID: 19474150

Sayama, K., K. Kajiya, K. Sugawara, S. Sato and S. Hirakawa et al., 2010. Inflammatory mediator TAK1 regulates hair follicle morphogenesis and anagen induction shown by using keratinocytespecific TAK1-deficient mice. PLoS One, 5: e11275-e11275. PMID: 20585657
Schneider, M.R., R. Schmidt-Ullrich and R. Paus, 2009. The hair follicle as a dynamic miniorgan. Curr. Biol., 19: R132-R142. PMID: 19211055

Shirakata, Y., 2010. Regulation of epidermal keratinocytes by growth factors. J. Dermatol. Sci., 59: 73-80. PMID: 20570492

Sriwiriyanont, P., A. Hachiya, W.L. Pickens, S. Moriwaki and T. Kitahara et al., 2011. Effects of IGF-binding protein 5 in dysregulating the shape of human hair. J. Invest. Dermatol., 131: 320-328. PMID: 20944648

Sugawara, K., M.R. Schneider, M. Dahlhoff, J.E. Kloepper and R. Paus, 2010. Cutaneous consequences of inhibiting EGF receptor signaling in vivo: Normal hair follicle development, but retarded hair cycle induction and inhibition of adipocyte growth in Egfr(Wa5) mice. J. Dermatol. Sci., 57: 155-161. PMID: 20060271

Tang, L.Y. and Y.E. Zhang, 2011. Non-degradative ubiquitination in Smad-dependent TGF- $\beta$ signaling. Cell Biosci., 1: 43-43. PMID: 22204598

Trusolino, L., A. Bertotti and P.M. Comoglio, 2010. MET signalling: Principles and functions in development, organ regeneration and cancer. Nat. Rev. Mol. Cell Biol., 11: 834-848. PMID: 21102609

Wilson, K.J., J.L. Gilmore, J. Foley, MA. Lemmon and D.J. Riese, 2009. Functional selectivity of EGF family peptide growth factors: implications for cancer. Pharmacol. Ther., 121: 1-8. PMID: 19135477

Xie, Y., K.J McElwee, G.R. Owen, L. Häkkinen and H.S. Larjava, 2012. Integrin $\beta 6$-deficient mice show enhanced keratinocyte proliferation and retarded hair follicle regression after depilation. J. Invest. Dermatol., 132: 547-555. PMID: 22113470

Xu, Y., W. Xia, D. Baker, J. Zhou and H.C. Cha et al., 2011. Receptor-type protein tyrosine phosphatase beta (RPTP-beta) directly dephosphorylates and regulates hepatocyte growth factor receptor (HGFR/Met) function. J. Biol. Chem., 286: 1598015988. PMID: 21454675

Yamamoto-Fukuda, T., H. Takahashi and T. Koji, 2012. Expression of Keratinocyte Growth Factor (KGF) and its receptor in a middle-ear cavity problem. Int. J. Pediatr. Otorhinolaryngol., 76: 7681. PMID: 22024578

Yang, C.C. and G. Cotsarelis, 2010. Review of hair follicle dermal cells. J. Dermatol. Sci., 57: 2-11. PMID: 20022473

Yoo, B.Y., Y.H. Shin, H.H. Yoon, Y.K. Seo and J.K. Park, 2010. Hair follicular cell/organ culture in tissue engineering and regenerative medicine. Biochem. Eng. J., 48: 323-331. DOI: 10.1016/j.bej.2009.09.008 
Zhang, D., G. Lijuan, L. Jingjie, L. Zheng and C. Wang et al., 2011. Cow placenta extract promotes murine hair growth through enhancing the insulin - like growth factor-1. Indian J. Dermatol., 56: 14-18. PMID: 21572784

Zhao, J., N. Harada, H. Kurihara, N. Nakagata and K. Okajim, 2011. Dietary isoflavone increases insulinlike growth factor-I production, thereby promoting hair growth in mice. J. Nutr. Biochem., 22: 227233. PMID: 20576422

Zhou, A.X., A. Toylu, R.K. Nallapalli, G. Nilsson and N. Atabey et al., 2011. Filamin a mediates HGF/cMET signaling in tumor cell migration. Int. J. Cancer, 128: 839-846. PMID: 20473907
Zhou, X. and Y.M. Agazie, 2012. The signaling and transformation potency of the overexpressed HER2 protein is dependent on the normally-expressed EGFR. Cell. Signal, 24: 140-150. PMID: 21911055

Zi, Z., D.A. Chapnick and X. Liu, 2012. Dynamics of TGF- $\beta /$ Smad signaling. FEBS Lett., 586: 19211928. PMID: 22710166

Zippel, J., T. Wells and A. Hensel, 2010. Arabinogalactan protein from Jatropha curcas L. seeds as TGF $\beta 1$-mediated inductor of keratinocyte in vitro differentiation and stimulation of GM-CSF, HGF, KGF and in organotypic skin equivalents. Fitoterapia, 81 : 772-778. DOI: 10.1016/j.fitote.2010.04.002 